\title{
On m-independence in Graphs
}

\author{
D. K. Thakkar ${ }^{1}$ and Neha P. Jamvecha ${ }^{2 *, 1}$ \\ ${ }^{1}$ Department of Mathematics, Saurashtra University, Rajkot-360005, Gujarat, India \\ ${ }^{2}$ Department of Mathematics, Saurashtra University, Rajkot-360005, Gujarat, India \\ *Corresponding Author: jamvechaneha30@gmail.com
}

Available online at: www.isroset.org

Accepted 17/Aug/2018, Online 30/Aug/2018

\begin{abstract}
In this paper, we have defined the concepts of $\mathrm{m}$-independent set, maximal $\mathrm{m}$-independent set and maximum $\mathrm{m}$ independent set. In order to define these concepts we have used the notion of $\mathrm{m}$-adjacent vertices. Adjacent vertices are always $\mathrm{m}$-adjacent vertices. This notion also gives rise to a concept called $\mathrm{m}$-domination in graphs. We prove that a set is maximal $\mathrm{m}$ independent set if and only if it is a minimal m-dominating set. We define m-independence number of a graph to be the maximum cardinality of an m-independent set. We prove a necessary and sufficient condition under which the m-independence number decreases when a vertex is removed from the graph. Further, we have also introduced a new operation in graph called $\mathrm{m}$-removal of a vertex. The subgraph obtained by m-removing a vertex is a subgraph of the subgraph obtained by removing the vertex from the graph. We prove that a vertex is an isolated vertex if and only if the m-independence number of the graph decreases when the vertex is m-removed from the graph. Some related examples have been given to illustrate these concepts.
\end{abstract}

Keywords - m-independent set, maximal m-independent set, maximum m-independent set, m-independence number, mdominating set, minimal $m$-dominating set, $m$-removal of a vertex

AMS Subject Classification-05C69

${ }^{1}$ The present research work for Neha P. Jamvecha is financially supported by INSPIRE FELLOWSHIP of Department of Science and Technology of Government of India.

\section{INTRODUCTION}

In the area of mixed domination, the concept of a vertex which m-dominates an edge and the concept of an edge which m-dominates a vertex have been defined an studied by some authors like R. Laskar, K. Peters, E. Sampathkumar, S. S. Kamath and others $[3,4,5]$. The above concepts can be used to define m-adjacent vertices and $\mathrm{m}$-adjacent edges. In fact, we have defined $\mathrm{m}$-adjacent vertices and $\mathrm{m}$-adjacent edges in [2]. Now we introduce a variant of (vertex) independence using the m-adjacency of vertices. We call a set $S$ of vertices to be an $\mathrm{m}$-independent set if no two distinct vertices of $S$ are m-adjacent. We also introduce maximal mindependent sets and maximum $\mathrm{m}$-independent sets in a similar way as we can do for maximal independent set. We have also a concept called m-domination in graphs which has been introduced in [1]. Maximum m-independent sets can also be related to $\mathrm{m}$-dominating sets.

A new operation called m-removal of a vertex has also been introduced here. We proved the effect of mremoving a vertex on $\mathrm{m}$-independence number.
The paper is organised as follows, Section I provides the introduction of the paper. Section II contains preliminaries and notations. Main results and Examples have been presented in Section III. Section IV provides conclusion of the paper also provides directions for the future research.

\section{Preliminaries AND Notations}

If $G$ is a graph then $E(G)$ denotes the edge set and $V(G)$ denotes the vertex set of the graph. If $v$ is a vertex of $G$ then $G \backslash v$ denotes the subgraph of $G$ obtained by removing the vertex $v$ and all the edges incident to $v . N(v)$ denotes the set of vertices which are adjacent to $v . N[v]=$ $N(v) \cup\{v\}$. If $x$ is any vertex then $d(x)$ denotes the degree of $x$ and is the number of edges incident at $x$. If $G$ is a graph then $\beta_{0}(G)$ denotes the independence number of a graph $G$.

\section{Main Results}

Definition 3.1: (m-vertex independent set) 
Let $G$ be a graph and $S \subset V(G)$ then $S$ is said to be an mvertex independent set or just $\mathrm{m}$-independent set if two distinct vertices of $S$ are m-adjacent.

It is obvious that every m-independent set is an independent set but independent set need not be an m-independent set.

Example 3.2: Consider cycle $C_{5}$ with vertices $\left\{v_{1}, v_{2}\right.$, $\left.v_{3}, v_{4}, v_{5}\right\}$

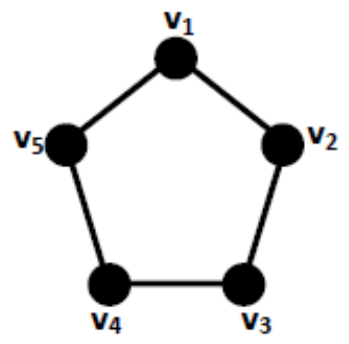

Figure 1. Cycle graph $C_{5}$

Let $S=\left\{v_{1}, v_{3}\right\}$ then $S$ is an independent set but not an $\mathrm{m}$ independent set.

Note that any subset of m-independent set is an $\mathrm{m}$ independent set but a super set of an m-independent set need not be an m-independent set. Thus m-independence is hereditary property but it is not super hereditary property.

Definition 3.3: (maximal m-independent set)

An m-independent set $F$ is said to be a maximal mindependent set if for every vertex $v$ not in $F, F \cup\{v\}$ is not an m-independent set.

Definition 3.4: (maximum m-independent set)

An m-independent set with maximum cardinality is called maximum $\mathrm{m}$-independent set. The cardinality of maximum m-independent set is denoted as $\beta_{m}(G)$ and is called the mindependence number of the graph $G$.

Example 3.5: Consider cycle $C_{5}$ with vertices $\left\{v_{1}, v_{2}\right.$, $\left.v_{3}, v_{4}, v_{5}\right\}$

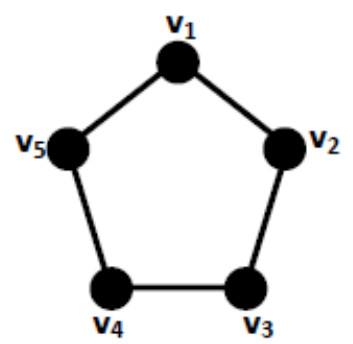

Figure 2. Cycle graph $C_{5}$ Figure 3.

Here, $\beta_{0}\left(C_{5}\right)=2$ and $\beta_{m}\left(C_{5}\right)=1$.
It is obvious that for any graph $G, \beta_{m}(G) \leq \beta_{0}(G)$.

Definition 3.6: (m-dominating set) [1]

Let $G$ be a graph and $S \subset V(G)$. Then $S$ is said to be an m-dominating set if for every vertex $v$ in $V(G) \backslash S$, there is a vertex $u$ in $S$ such that $u$ and $v$ are m-adjacent.

Definition 3.7: (minimal m-dominating set) [1]

Let $G$ be a graph and $S \subset V(G)$ be an m-dominating set. Then $S$ is said to be a minimal m-dominating set if $S \backslash\{v\}$ is not an m-dominating set for every $v$ in $S$.

Theorem 3.8: Let $G$ be a graph and $S \subset V(G)$ be an mindependent set. Then $S$ is a maximal m-independent set if and only if $S$ is an m-dominating set.

Proof: Suppose $S$ is a maximal m-independent set. Let $v \in V(G) \backslash S$. Now, $S \cup\{v\}$ is not an m-independent set. There are two vertices of $S \cup\{v\}$ which are m-adjacent.

Since $S$ is an m-independent set, one of these vertex must be $v$. Thus, $v$ is m-adjacent with some vertex of $S$. Thus $S$ is an m-dominating set.

Conversely, suppose $S$ is an m-dominating set. Let $v \in V(G) \backslash S$. Then there is a vertex $u \in S$ such that $u$ and $v$ are m-adjacent. Thus there are vertices in $S \cup\{v\}$ which are m-adjacent. Thus, $S$ is a maximal $\mathrm{m}$-independent set.

Now we prove a stronger result then the above theorem.

Theorem 3.9: Let $G$ be a graph and $S \subset V(G)$ be an mindependent set. Then $S$ is a maximal m-independent set if and only if $S$ is a minimal m-dominating set.

Proof: Suppose, $S$ is a maximal m-independent set. We have already proved that $S$ is an m-dominating set. Let $v \in S$ and consider $S_{1}=S \backslash\{v\}$. Now, $v \notin S_{1}$. Also $v$ is not m-adjacent to any vertex of $S$ because $S$ is an mdominating set. Thus $v$ is not $\mathrm{m}$-adjacent to any vertex of $S_{1}$. This means that $S_{1}$ is not an m-dominating set. Since $v \in S$ is arbitrary it follows that $S$ is a minimal mdominating set.

Conversely, suppose $S$ is a minimal m-dominating set. Since $S$ is an m-independent set and an m-dominating set, it is a maximal m-independent set by the theorem 3.8. Thus the theorem is proved.

We know that for any graph $G$ and $v \in V(G)$, $\beta_{0}(G \backslash v) \leq \beta_{0}(G)$. However, the similar result need not be true for $\mathrm{m}$-independence.

Consider the following examples.

Example 3.10: Consider cycle $C_{6}$ with vertices $\left\{v_{1}, v_{2}\right.$, $\left.v_{3}, v_{4}, v_{5}, v_{6}\right\}$ 


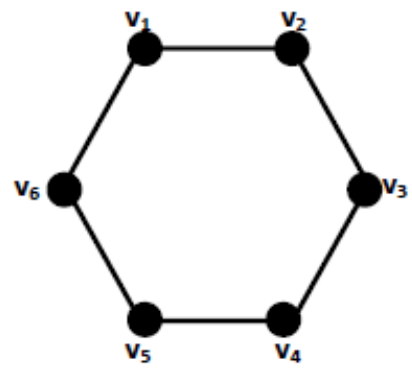

Figure 4. Cycle graph $C_{6}$

Let $M=\left\{v_{1}\right\}$. It is obvious that $M$ is a maximum mindependent set of $C_{6}$ and therefore $\beta_{m}\left(C_{6}\right)=1$. Now consider the vertex $v_{6}$ and consider the subgraph $C_{6} \backslash\left\{v_{6}\right\}$, which is a path graph with vertices $\left\{v_{1}, v_{2}, v_{3}, v_{4}, v_{5}\right\}$. In this graph $M_{1}=\left\{v_{1}, v_{5}\right\}$ is a maximum m-independent set of $C_{6} \backslash\left\{v_{6}\right\}$.

Therefore, $\beta_{m}\left(C_{6} \backslash v_{6}\right)=2$ and $\beta_{m}\left(C_{6} \backslash v_{6}\right)>\beta_{m}\left(C_{6}\right)$.

Again consider the following example.

Example 3.11: Consider the path graph $P_{9}$ with vertices $\left\{v_{1}, v_{2}, v_{3}, v_{4}, v_{5}, v_{6}, v_{7}, v_{8}, v_{9}\right\}$

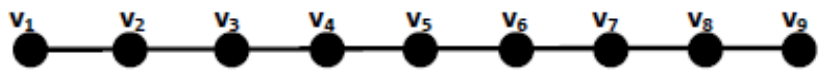

Figure 5. Path graph $P_{9}$

Then $\beta_{m}\left(P_{9} \backslash v_{5}\right)<\beta_{m}\left(P_{9}\right)$ and $\beta_{m}\left(P_{9} \backslash v_{4}\right)=\beta_{m}\left(P_{9}\right)$.

Proposition 3.12: Let $v$ be a pendant vertex then $\beta_{m}(G \backslash v) \leq \beta_{m}(G)$.

Proof: Let $M$ be a maximum m-independent set of $G \backslash v$. Then $d(x, y) \geq 4$ in $G \backslash v$ for any $\{x, y\} \in \mathbf{M}$. Since $v$ is a pendant vertex, there is no path between $x$ and $y$ which includes $v$. Therefore, every path between $x$ and $y$ in $G$ is also a path in $G \backslash v$. Therefore, $d(x, y)$ in $G$ is equal to $d(x, y)$ in $G \backslash v$. Thus $d(x, y) \geq 4$ in $G$ for any $\{x, \mathrm{y}\} \in \mathrm{M}$. Therefore, $M$ is an m-independent subset of $G$. Therefore, $\beta_{m}(G) \geq|M|=\beta_{m}(G \backslash v)$.

Theorem 3.13: Let $G$ be a graph and $v \in V(G)$. Then $\beta_{m}(G \backslash v)<\beta_{m}(G)$ if and only if following two conditions are satisfied

(i) There is a maximum m-independent set $M$ of $G \backslash v$ such that $M$ is also m-independent set in $G$.

(ii) $v$ is not m-adjacent with any vertex of $M$ in $G$.

Proof: Suppose $\beta_{m}(G \backslash v)<\beta_{m}(G)$.
Claim: there is a maximum m-independent set $M_{1}$ of $G$ such that $v \in M_{1}$.

Proof of the claim: Suppose there is no such set $M_{1}$. Let $M_{1}$ be any maximum m-independent set of $G$. Then $v \notin M_{1}$. Obviously, $M_{1}$ is an m-independent set of $G \backslash v$. Therefore, $\beta_{m}(G \backslash v) \geq\left|M_{1}\right|=\beta_{m}(G)$ this is a contradiction. Thus, there is a maximum m-independent set $M_{1}$ of $G$ such that $v \in M_{1}$. Let $M=M_{1} \backslash\{v\}$. Then $M$ is a maximum mindependent set of $G \backslash v$ because $\beta_{m}(G \backslash v)<\beta_{m}(G)$. Thus condition (i) is satisfied.

Since $v \in M_{1}$ and $M_{1}$ is an m-independent set, $v$ is not $\mathrm{m}$ adjacent with any other vertex of $M_{1}$. This means that $v$ is not m-adjacent to any vertex of $M$. Thus condition (ii) is also satisfied.

Conversely, suppose condition (i) and (ii) are satisfied.

Let $M$ be a maximum m-independent set of $G \backslash v$ which is also an m-independent set of $G$. And suppose $v$ is not $\mathrm{m}$ adjacent to any vertex of $M$ in $G$. Let $M_{1}=M \cup\{v\}$ then $M_{1}$ is an m-independent set of $G$. Therefore, $\beta_{m}(G) \geq\left|M_{1}\right|>|M|=\beta_{m}(G \backslash v)$. Thus $\beta_{m}(G \backslash v)<\beta_{m}(G)$.

Theorem 3.14: Let $G$ be a graph and $v \in V(G)$. If $\beta_{m}(G \backslash v)<\beta_{m}(G)$ then for every maximum m-independent set $M$ of $G, v \in M$.

Proof: Suppose there is a maximum m-independent set $M$ of $G$ such that $v \notin M$. Then $M$ is an m-independent set in $G \backslash v$. Therefore, $\beta_{m}(G \backslash v) \geq|M|=\beta_{m}(G)$ which contradicts the hypothesis that $\beta_{m}(G \backslash v)<\beta_{m}(G)$. Thus $v \in M$ for every maximum m-independent set $M$ of $G$.

Remark 3.15: The condition mentioned in the theorem 3.14 is necessary but not sufficient.

Consider the following example

Example 3.16: Consider the graph $G$ whose vertices are $\left\{v_{1}, v_{2}, v_{3}, v_{4}, v_{5}, v_{6}, v_{7}, v_{8}\right\}$

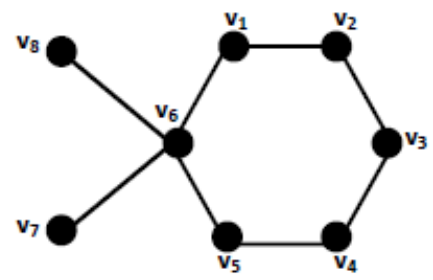

Figure 6. Graph $G$

The only maximum m-independent sets of this graph are $M_{1}=\left\{v_{3}, v_{7}\right\}$ and $M_{2}=\left\{v_{3}, v_{8}\right\}$. The vertex $v_{3} \in$ Every 
maximum m-independent subset of $G$. If we remove vertex $v_{3}$ then $\beta_{m}\left(G \backslash v_{3}\right)=2$ which is $\left\{v_{2}, v_{4}\right\}$.

Corollary 3.17: Let $G$ be a graph and $M$ be a maximum m-independent subset of $G$. Let $v \in V(G) \backslash M$ then $\beta_{m}(G \backslash v) \geq \beta_{m}(G)$.

Proof: Suppose $v \in V(G) \backslash M$.

Since $v \notin M, \beta_{m}(G \backslash v)<\beta_{m}(G)$ which is not possible. Therefore, $\beta_{m}(G \backslash v) \geq \beta_{m}(G)$.

Theorem 3.18: Let $G$ be a graph and $v \in V(G)$. If $\beta_{m}(G \backslash v)>\beta_{m}(G)$ then for every maximum m-independent subset $M$ of $G \backslash v$, there are vertices $x, y$ in $M$ such that at least one of the following two conditions holds.

(i) $x$ is adjacent to $v$ and $d(v, y) \leq 2$ in $G$.

(ii) $y$ is adjacent to $v$ and $d(v, x) \leq 2$ in $G$.

Proof: Suppose $\beta_{m}(G \backslash v)>\beta_{m}(G)$. Let $M$ be any maximum m-independent subset of $G \backslash v$. Then $M$ cannot be an m-independent subset of $G$ because it would imply that $\beta_{m}(G) \geq|M|=\beta_{m}(G \backslash v)$. Since $M$ is not an mindependent subset of $G$, there are vertices $\{x, y\} \in M$ such that $x$ and $y$ are m-adjacent in $G$ (But $x$ and $y$ are not m-adjacent in $G \backslash v)$. Therefore, $d(x, y) \leq 3$ in $G$ but $d(x, y) \geq 4$ in $G \backslash v$. Therefore, the shortest path joining $x$ and $y$ in $G$ must contain $v$.

If $d(x, y)=2$ in $G$ then $x$ and $y$ are adjacent to $v$ and condition (i) and (ii) both are satisfied.

If $d(x, y)=3$ in $G$ then there is an edge $u v$ such that $x$ is adjacent to $v$ and $y$ is adjacent to $u$ or $x$ is adjacent to $u$ and $y$ is adjacent to $v$. In the first case condition (i) is satisfied and in the second case condition (ii) is satisfied. It is not possible that both $x$ and $y$ are adjacent to $u$ because it would imply that $d(x, y)=2$ in $G \backslash v$ which is not.

Thus, the statement is true.

Remark 3.19: Let $P_{n}$ be the path with $n$-vertices.

(i) If $n=4 k+1$ for some $k$ then $P_{n}$ has a maximum mindependent set with $k+1$ vertices and for each end vertex $v, \beta_{m}\left(P_{n} \backslash v\right)<\beta_{m}\left(P_{n}\right)$.

(ii) If $n=4 k+2$ or $4 k+3$, then also $P_{n}$ has a maximum mindependent set of having $k+1$ vertices and for every pendant vertex $v, \beta_{m}\left(P_{n} \backslash v\right)=\beta_{m}\left(P_{n}\right)$.

(iii) If $n=4 k$ for some $k$ then $P_{n}$ has a maximum mindependent set with $k$ vertices and for every pendant vertex $v, \beta_{m}\left(P_{n} \backslash v\right)=\beta_{m}\left(P_{n}\right)$.
We now introduce a new operation in graph called mremoval of vertex.

Definition 3.20: (m-removal of a vertex)

Let $G$ be a graph and $v \in V(G)$. We obtain a subgraph of $G$ by removing a vertex $v$ and certain edges which will be called the subgraph obtained by m-removing the vertex $v$ from the graph $G$.

Definition 3.21: Let $G$ be a graph and $v \in V(G)$. The subgraph obtained by m-removing vertex $v$ from $G$ has the vertex set $V(G) \backslash\{v\}$ and by removing all the edges of $G$ which m-dominate vertex $v$. This subgraph is denoted as $G \backslash^{m}\{v\}$.

Example 3.22: Consider the cycle graph $C_{5}$ with vertices $\left\{v_{1}, v_{2}, v_{3}, v_{4}, v_{5}\right\}$

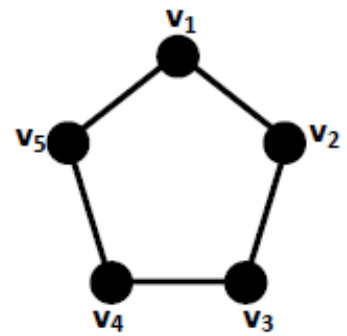

Figure 7. Cycle graph $C_{5}$

Consider the vertex $v_{5}$. Then the vertices of $G \backslash^{m}\left\{v_{5}\right\}$ are $\left\{v_{1}, v_{2}, v_{3}, v_{4}\right\}$ and it has only one edge namely $\left\{v_{2} v_{3}\right\} \cdot v_{1}$ and $v_{4}$ are isolated vertices in this subgraph.

Note that $G \backslash^{m}\{v\}$ is subgraph of $G \backslash\{v\}$.

Remark 3.23: Let $G$ be a graph and $v \in V(G)$.

(i) Let $x, y \in V(G)$ such that $x \neq v$ and $y \neq v$ then $d(x, y)$ in $G \backslash^{m}\{v\} \geq d(x, y)$ in $G$. In particular, if $x$ and $y$ are not m-adjacent in $G$. Then $x$ and $y$ are not m-adjacent in $G \backslash^{m}\{v\}$.

(ii) If $v$ is an isolated vertex in $G$ then $d(v, u)>k$ for every positive integer $k$ and $u \neq v$. In particular, $d(v, u) \geq 4$, for any other vertex $u$ of $G$. Also if $x \neq v$ and $y \neq v$ then $d(x, y)$ in $G \backslash^{m}\{v\}=d(x, y)$ in $G$.

(iii) If $v$ is not an isolated vertex then every neighbor $u$ of $v$ is an isolated vertex in $G \backslash^{m}\{v\}$.

Proposition 3.24: Let $G$ be a graph and $v$ be a non-isolated vertex of $G$. Then $\beta_{m}\left(G \backslash^{m}\{v\}\right) \geq \beta_{m}(G)$. 
Proof: Let $M$ be a maximum m-independent subset of $G$. Case-(i) $v \in M$

Let $u$ be a neighbor of $v$ in $G$. Then $u \notin M$ and $u$ is an isolated vertex in $G \backslash^{m}\{v\}$. Now let $M_{1}=(M \backslash\{v\}) \cup\{u\}$.

Then $M_{1}$ is an m-independent subset of $G \backslash^{m}\{v\}$. Therefore, $\beta_{m}\left(G \backslash^{m}\{v\}\right) \geq\left|M_{1}\right|=|M|=\beta_{m}(G)$.

Case-(ii) $v \notin M$

First assume that all the vertices which are m-adjacent to $v$ are in $M$. Then, $M$ is an m-independent subset in $G \backslash^{m}\{v\}$ also. Therefore, $\beta_{m}\left(G \backslash^{m}\{v\}\right) \geq \beta_{m}(G)$.

Suppose there is a vertex $u$ which is m-adjacent to $v$ and $u \notin M$. Then $u$ is an isolated vertex in $G \backslash^{m}\{v\}$ by the remark 3.23. Let $M_{1}=M \cup\{u\}$. Then $M_{1}$ is an mindependent subset of $G \backslash^{m}\{v\}$.Therefore, $\beta_{m}\left(G \backslash^{m}\{v\}\right) \geq$ $\left|M_{1}\right|>|M|=\beta_{m}(G)$.

From both the cases it follows that $\beta_{m}\left(G \backslash^{m}\{v\}\right) \geq \beta_{m}(G)$.

Proposition 3.25: Let $G$ be a graph and $v$ be a vertex of $G$. Then $v$ is an isolated vertex if and only if $\beta_{m}\left(G \backslash^{m}\{v\}\right)<\beta_{m}(G)$.

Proof: Suppose, $v$ is an isolated vertex of $G$. Let $M$ be a maximum m-independent subset of $G \backslash^{m}\{v\}$. If $x, y \in M$. Then $d(x, y) \geq 4$ in $G$. Therefore, $x$ and $y$ are non $\mathrm{m}-$ adjacent vertices in $G$. Therefore, $M$ is an m-independent subset of $G$.

Therefore, $\beta_{m}(G) \geq|M \cup\{v\}|>|M|=\beta_{m}\left(G \backslash^{m}\{v\}\right)$.

Therefore, $\beta_{m}\left(G \backslash^{m}\{v\}\right)<\beta_{m}(G)$.

Conversely, Suppose $\beta_{m}\left(G \backslash^{m}\{v\}\right)<\beta_{m}(G)$. If $v$ is not an isolated vertex of $G$ then by proposition 3.24 $\beta_{m}\left(G \backslash^{m}\{v\}\right) \geq \beta_{m}(G)$, which is a contradiction. Therefore, $v$ is an isolated vertex of $G$.

Corollary 3.26: Let $G$ be a graph and $v$ is an isolated vertex of $G$. Then $\beta_{m}(G)=\beta_{m}\left(G \backslash^{m}\{v\}\right)+1$.

Proof: Let $M$ be a maximum m-independent subset of $G$. Then $v \in M$. Let $M_{1}=M \backslash\{v\}$. Then, $M_{1}$ is an $\mathrm{m}-$ independent subset of $G \backslash^{m}\{v\}$. Since $\beta_{m}\left(G \backslash^{m}\{v\}\right)<$ $\beta_{m}(G), M_{1}$ is a maximum m-independent subset of
$G \backslash^{m}\{v\}$. Therefore, $\beta_{m}\left(G \backslash^{m}\{v\}\right)=\left|M_{1}\right|=|M|-1=\beta_{m}(G)$ -1 . Therefore, $\beta_{m}(G)=\beta_{m}\left(G \backslash^{m}\{v\}\right)+1$.

Corollary 3.27: Let $G$ be a graph and $v$ is an isolated vertex of $G$. Then

(i) There is a one-one correspondence between the maximum m-independent subsets of $G$ and the maximum mindependent subsets of $G \backslash^{m}\{v\}$.

(ii) The number of maximum m-independent subsets of $G$ is equal to the number of maximum m-independent subsets of $G \backslash^{m}\{v\}$.

Proof: If $M$ is a maximum m-independent subset of $G \backslash^{m}\{v\}$ then by the corollary 3.26, $M \cup\{v\}$ is a maximum m-independent subset of $G$. If $M_{1}$ and $M_{2}$ are distinct mindependent subset of $G \backslash^{m}\{v\}$. Then $M_{1} \cup\{v\} \neq M_{2} \cup\{v\}$. If $M^{\prime}$ is a maximum m-independent subset of $G$ and if $M=M^{\prime} \backslash\{v\}$ then $M$ is a maximum m-independent subset of $G \backslash^{m}\{v\}$ and $M^{\prime}=M \cup\{v\}$. Thus, $M \rightarrow M \cup\{v\}$ is a one-one correspondence between the set of all maximum mindependent subsets of $G \backslash^{m}\{v\}$ and the set of all maximum m-independent subsets of $G$. Thus (i) is established.

Similarly, (ii) is obvious.

\section{CONCLUSION AND Future SCOPE}

We have proved a necessary condition under which the mindependence number increases when a vertex is removed from the graph. It is interesting to know what is sufficient condition under which the $\mathrm{m}$-independence number increases when a vertex is removed from the graph. It may be further investigated how the m-independence number is affected when an edge is removed or added for the given graph.

\section{ACKNOWLEDGMENT}

The Author would like to acknowledge their thanks to the Editor-in-chief and Referees for their valuable suggestions to improve the quality of this paper.

\section{REFERENCES}

[1] D. K. Thakkar and Neha P. Jamvecha, "About m-domination number of Graphs" (communicated).

[2] D. K. Thakkar and Neha P. Jamvecha, "A New Variant of Edge Stability in Graph", International Journal of Pure and Engineering Mathematics, Vol.5, Issue.3, pp.87-97, 2017.

[3] R. Laskar and K. Peters, "Vertex and edge domination parameters in graphs", Congressus Numerantium, Vol.48, pp.291-305, 1985.

[4] E. Sampathkumar, and P. S. Neeralagi, "The neighbourhood number of a graph", Journal of Pure and Applied Mathematics, Vol.16, Issue.2, pp.126-136, 1985. 
[5] E. Sampathkumar, and S. S. Kamath, "Mixed Domination in Graphs", The Indian Journal of Statistics, 1992.

[6] T. W. Haynes, S. T. Hedetniemi and P. J. Slater, "Domination in Graphs Advanced Topics”, Marcel Dekker, Inc., New-York, 1998.

[7] T. W. Haynes, S. T. Hedetniemi, and P. J. Slater, "Fundamentals of domination in graphs", Marcel Dekker, Inc., New-York, 1998.

\section{AUTHORS PROFILE}

Dr. D. K. Thakkar is a retired professor of Mathematics, Saurashtra University, Rajkot. His areas of interest are Graph Theory and Topology. He has published more than 85 research papers in various journals.

Ms Neha P. Jamvecha is a research student who is working in the area of Mixed Domination in graphs. She is an INSPIRE Fellow in Department of Mathematics, Saurashtra University, Rajkot. 